\title{
A complex-angle rotation and geometric complementarity in fermion mixing
}

\author{
Kee-Hwan Nam* and Kim Siyeont \\ Department of Physics, Chung-Ang University, Seoul 156-756, Korea \\ Seungsu Hwang $\ddagger$ \\ Department of Mathematics, Chung-Ang University, Seoul 156-756, Korea
}

(Dated: today)

\begin{abstract}
The mixing among flavors in quarks or leptons in terms of a single rotation angle is defined such that three flavor eigenvectors are transformed into three mass eigenvectors by a single rotation about a common axis. We propose that a geometric complementarity condition exists between the complex angle of quarks and that of leptons in $\mathbb{C}^{2}$ space. The complementarity constraint has its rise in quark-lepton unification and is reduced to the correlation among $\theta_{12}, \theta_{23}, \theta_{13}$ and the $\mathrm{CP}$ phase $\delta$. The CP phase turns out to have a non-trivial dependence on all the other angles. We will show that further precise measurements in real angles can narrow down the allowed region of $\delta$. In comparison with other complementarity schemes, this geometric one can avoid the problem of the $\theta_{13}$ exception and can naturally keep the lepton basis being independent of quark basis.
\end{abstract}

PACS numbers: 11.30.Fs, 14.60.Pq, 14.60.St

Keywords: neutrino mixing, quark-lepton complementarity

\footnotetext{
*Electronic address: snowall@gmail.com

${ }^{\dagger}$ Electronic address: siyeon@cau.ac.kr

${ }^{\ddagger}$ Electronic address: seungsu@cau.ac.kr
} 


\section{INTRODUCTION}

With a number of successful neutrino oscillation experiments, the information on fermion masses and on the transformation between the mass basis and the weak interaction basis is getting more balanced between the quark part and the lepton part. The Cabibbo-KobayashiMaskawa(CKM) matrix of quark mixing is just a few steps from the completion. The allowed ranges of the magnitudes of the CKM elements are narrow, $\left|V_{C K M}\right|=$

$$
\left(\begin{array}{ccc}
0.97383_{-0.00023}^{+0.00024} & 0.2272_{-0.0010}^{+0.0010} & \left(3.96_{-0.09}^{+0.09}\right) \times 10^{-3} \\
0.2271_{-0.0010}^{+0.0010} & 0.97296_{-0.00024}^{+0.00024} & \left(42.21_{-0.80}^{+0.10}\right) \times 10^{-3} \\
\left(8.14_{-0.64}^{+0.32}\right) \times 10^{-3} & \left(41.61_{-0.78}^{+0.12}\right) \times 10^{-3} & 0.999100_{-0.000004}^{+0.000034}
\end{array}\right),
$$

while the ranges of the magnitudes of Potecorvo-Maki-Nakagawa-maskawa(PMNS) elements are still broad;

$$
\left|U_{P M N S}\right|=\left(\begin{array}{ccc}
0.79-0.86 & 0.50-0.61 & 0-0.20 \\
0.25-0.53 & 0.47-0.73 & 0.56-0.79 \\
0.21-0.51 & 0.42-0.69 & 0.61-0.83
\end{array}\right)
$$

at the $3 \sigma$ level [1]. The unitary transformations are conventionally described as Euler-type subsequent operations of three separate rotations,

$$
U\left(\theta_{23}, \theta_{13}, \theta_{12}, \delta\right)=R\left(\theta_{23}\right) R\left(\theta_{13}, \delta\right) R\left(\theta_{12}\right)
$$

that is, through a rotation in the $1-2$ plane, another rotation in the $1^{\prime}-3$ plane, and a third rotation in the $2^{\prime}-3^{\prime}$ plane, the mass basis is switched into the weak basis. From an analysis of the global data, the three angles in CKM have the best-fit values $\theta_{12}^{q}=0.229, \theta_{13}^{q}=0.004$, and $\theta_{23}^{q}=0.042$, and the angles in PMNS have the best-fit values $\theta_{12}^{l}=0.588, \theta_{13}^{l}=0$, and $\theta_{23}^{l}=0.756[2,3]$.

Quark-lepton complementarity (QLC) is one of the theoretical frameworks on which phenomenological data can be naturally connected to the quark-lepton unification. In many works, the QLC idea was built by the relation [4]

$$
\theta_{i j}^{q}+\theta_{i j}^{l}=\frac{\pi}{4}
$$

with the mixing angle between $\mathrm{i}$ - and $\mathrm{j}$ - generations, $\theta_{i j}$. Only $\theta_{12}$ 's and $\theta_{23}$ 's satisfy the above relation such that the complementarity gives rise to bi-maximal mixing. 
The QLC represented by Eq. (3) has a few points unexplained so far. First, like bimaximal mixing, the QLC is obliged to keep the exception with small $\theta_{13}$ 's of quarks and leptons, which cannot make the sum maximal. Second, the relation implies that the angles $\theta_{i j}^{q}$ and $\theta_{i j}^{l}$ are in a plane. In low-energy theory where the quark-lepton symmetry is broken, the common plane including those two angles requires a strong generic connection between quark bases and lepton bases in process of symmetry breaking, but there is no supporting theory.

Here, we propose a model that accommodates small $\theta_{13}$ 's and that allows $\theta_{i j}^{q}$ and $\theta_{i j}^{l}$ to belong to independent planes. In this attempt, the transformation from the weak basis to the mass basis can be obtained by a single complex-angle rotation about a properly defined axis [5]. In other words, by a single rotation about an axis, the weak eigenstates $\left(\nu_{e}, \nu_{\mu}, \nu_{\tau}\right)$ or $(d, s, b)$ are switched into $\left(\nu_{1}, \nu_{2}, \nu_{3}\right)$ or $\left(d_{1}, d_{2}, d_{3}\right)$, respectively. Thus, there are two complex angles, one corresponding to quark mixing and the other corresponding to lepton mixing. We introduce the complementarity by a relation of those two complex angles such that

$$
\Theta_{L}^{2}-\Theta_{Q}^{2}=\left(\frac{\pi}{4}\right)^{2}
$$

where they are the orthogonal components of a hyperbola of radius $\pi / 4$. With such a geometric constraint, the model can protect the complementarity from the $\theta_{13}$ exception. Furthermore, the quark basis and the lepton basis are independent of each others as implied by completely broken quark-lepton symmetry.

In Section II, the definition of the complex angle and the axis to represent the transformation will be introduced. In Section III, using the hyperbolic condition, the allowed range of Dirac Charge-Parity violating $(\mathrm{CP})$ phase will be predicted. It will be shown that the more precise measurements in other angles in the future can test the model itself, as well as the $\mathrm{CP}$ violation testable in neutrino oscillation. Only the CKM-type matrix without Majorana phases is considered as the PMNS matrix. An extended work with more details, including Majorana phases and the physical implication relevant to them, is in progress in other work. A brief on the convention to deal with complex angles is attached. 


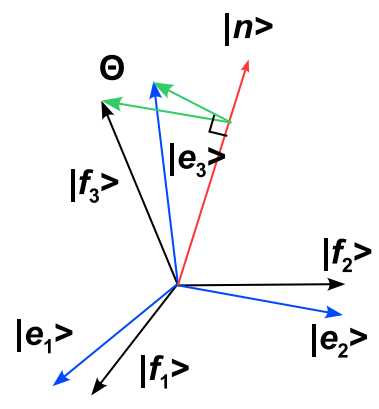

FIG. 1: The rotation of $\Theta$ about $\mid n>$ transforms $\mid e_{i}>$ 's into $\mid f_{\alpha}>$ 's.

\section{A SINGLE COMPLEX-ANGLE ROTATION}

In both quarks and leptons, a unitary transformation between flavor eigenstates $\left|f_{\alpha}\right\rangle$ and mass eigenstates $\left|e_{i}\right\rangle$ consists minimally of three angles and a CP phase:

$$
\left|f_{\alpha}\right\rangle=U\left(\theta_{12}, \theta_{13}, \theta_{23}, \delta\right)\left|e_{i}\right\rangle
$$

If the weak interaction basis is properly chosen such that the mass matrix of up-type quarks and that of charged leptons are diagonal, $\left|f_{\alpha}\right\rangle$ and $U$ in Eq. (5) represent either down-type quarks and the CKM matrix in the quark sector or neutrinos and the PMNS matrix in the lepton sector. Suppose, in a representation, that $\left|e_{1}\right\rangle=(1,0,0)^{T},\left|e_{2}\right\rangle=(0,1,0)^{T}$, and $\left|e_{3}\right\rangle=(0,0,1)^{T}$. Then $\left|f_{\alpha}\right\rangle=\left(U_{f_{\alpha} 1}, U_{f_{\alpha} 2}, U_{f_{\alpha} 3}\right)^{T}$, for $f_{\alpha}=d, s, b$ or $f_{\alpha}=e, \mu, \tau$. The $U_{f_{\alpha} i}$ is

an element of the transformation matrix in Eq. (5). The components of $f_{\alpha}$ are denoted by $\left(f_{1}, f_{2}, f_{3}\right)$ if the specification of 'quark or lepton' is not necessary. In a real vector space, an orthogonal set of three vectors can fit into another orthogonal set of three vectors simply by rotating the original set about a common axis, which can be found to be invariant under the rotation. Likewise, the unitary transformation in Eq. (5) can be replaced by a rotation with a single complex angle.

If one constructs a vector $|n\rangle$ as the axis of the rotation, the rotation angle of a vector $\left|e_{i}\right\rangle$ about $|n\rangle$ is the same as the angle between the following two vectors $\left|e_{i}^{\perp}\right\rangle$ and $\left|f_{\alpha}^{\perp}\right\rangle$ that are orthogonal to $|n\rangle$ :

$$
\begin{aligned}
\cos \Theta & =\frac{\left\langle e_{i}^{\perp} \mid f_{\alpha}^{\perp}\right\rangle}{\sqrt{\left\langle e_{i}^{\perp} \mid e_{i}^{\perp}\right\rangle} \sqrt{\left\langle f_{\alpha}^{\perp} \mid f_{\alpha}^{\perp}\right\rangle}} \\
& =\frac{\left\langle e_{i} \mid f_{\alpha}\right\rangle-\left\langle e_{i} \mid n\right\rangle\left\langle n \mid f_{\alpha}\right\rangle}{\sqrt{\left\langle e_{i}^{\perp} \mid e_{i}^{\perp}\right\rangle} \sqrt{\left\langle f_{\alpha}^{\perp} \mid f_{\alpha}^{\perp}\right\rangle}},
\end{aligned}
$$


where

$$
\begin{aligned}
& \left|f_{\alpha}^{\perp}\right\rangle=\left|f_{\alpha}\right\rangle-|n\rangle\left\langle n \mid f_{\alpha}\right\rangle, \\
& \left|e_{i}^{\perp}\right\rangle=\left|e_{i}\right\rangle-|n\rangle\left\langle n \mid e_{i}\right\rangle .
\end{aligned}
$$

The rotation axis $|n\rangle=\left(n_{x}, n_{y}, n_{z}\right)^{T}$ has the same components on the mass basis $\left|e_{i}\right\rangle$ as on the flavor basis $\left|f_{\alpha}\right\rangle$, that is,

$$
n_{x}\left|e_{1}\right\rangle+n_{y}\left|e_{2}\right\rangle+n_{z}\left|e_{3}\right\rangle=n_{x}\left|f_{1}\right\rangle+n_{y}\left|f_{2}\right\rangle+n_{z}\left|f_{3}\right\rangle
$$

which is a normalized vector with $\left|n_{x}\right|^{2}+\left|n_{y}\right|^{2}+\left|n_{z}\right|^{2}=1$. Substituting Eq. (5) into Eq. (7) results in the following combined equations:

$$
\begin{aligned}
& c_{13} c_{12} n_{x}-c_{13} s_{12} n_{y}+s_{13} e^{-i \delta} n_{z}=n_{x} \\
& \left(-c_{23} s_{12}-s_{23} s_{13} c_{12} e^{i \delta}\right) n_{x}+\left(c_{23} c_{12}-s_{23} s_{13} s_{12} e^{i \delta}\right) n_{y}+s_{23} c_{13} n_{z}=n_{y} \\
& \left(s_{23} s_{12}-c_{23} s_{13} c_{12} e^{i \delta}\right) n_{x}+\left(-s_{23} c_{12}-c_{23} s_{13} s_{12} e^{i \delta}\right) n_{y}+s_{23} c_{13} n_{z}=n_{z} .
\end{aligned}
$$

It is possible to express $|n\rangle$ immediately in terms of mixing parameters. For instance, $\left|e_{1}^{\perp}\right\rangle,\left|f_{1}^{\perp}\right\rangle$, the projected vectors of $\left|e_{1}\right\rangle,\left|f_{1}\right\rangle$ on the plane perpendicular to $|n\rangle$, are, according to Eq. (6),

$$
\begin{aligned}
&\left|e_{1}^{\perp}\right\rangle=\left(\begin{array}{c}
1-\left|n_{x}\right|^{2} \\
-n_{x}^{*} n_{y} \\
-n_{x}^{*} n_{z}
\end{array}\right), \\
&\left|f_{1}^{\perp}\right\rangle=\left(\begin{array}{c}
U_{11} \\
U_{12} \\
U_{13}
\end{array}\right)-\left(n_{x}^{*} U_{11}+n_{y}^{*} U_{12}+n_{z}^{*} U_{13}\right)\left(\begin{array}{c}
n_{x} \\
n_{y} \\
n_{z}
\end{array}\right) .
\end{aligned}
$$

Then, the $\cos \Theta$ in Eq. (마) reduces to

$$
\cos \Theta=\frac{U_{11}-n_{x}\left(n_{x}^{*} U_{11}+n_{y}^{*} U_{12}+n_{z}^{*} U_{13}\right)}{\left.\sqrt{\left(1-\left|n_{x}\right|^{2}\right)\left(1-\left|n_{x}^{*} U_{11}+n_{y}^{*} U_{12}+n_{z}^{*} U_{13}\right|^{2}\right.}\right)},
$$

because $\left(n_{x}, n_{y}, n_{z}\right)$ is obtained in terms of mixing angles and a phase, as is $\cos \Theta$.

The four physical parameters in the unitary mixing in Eq. (5) are now all embedded in the direction of $|n\rangle$. For a complex vector, one can remove the imaginary phase in one of the 
elements by multiplying all the elements in the vector by an overall phase factor. Together with normalization, the complex vector $|n\rangle=\left(n_{x}, n_{y}, n_{z}\right)^{T}$ does include just four independent parameters while the complex angle $\Theta$ does not include any additional independent parameter. Once $|n\rangle$ is found, then a unique $\Theta$ is determined to represent the transformation. It can be also checked that $\cos \Theta$ is complex unless $\sin \delta$ vanishes. As an example to find $|n\rangle$ and $\Theta$, if one considers $\theta_{12}=0.23, \theta_{23}=0.042, \theta_{13}=0.004$, and $\delta=0.99$ induced from the best-fit values of the elements in the CKM matrix, the rotation of an angle $\Theta=0.23 e^{i \varphi}, \varphi<0.01$, obtained from Eq. (10) about the axis

$$
\begin{aligned}
\left|n_{Q}\right\rangle & =0.18 e^{-.005 i}\left|e_{1}\right\rangle+0.017 e^{0.868 i}\left|e_{2}\right\rangle+0.983\left|e_{3}\right\rangle \\
& =0.18 e^{-.005 i}|d\rangle+0.017 e^{0.868 i}|s\rangle+0.983|b\rangle
\end{aligned}
$$

results in the same transformation as the CKM matrix does.

\section{HYPERBOLIC COMPLEMENTARITY CONDITION IN $\mathbb{C}^{2}$ SPACE}

As shown in the previous section, there exists a single complex angle rotation that replaces any three-dimensional unitary transformation. The single complex angles to replace CKM matrix in quarks and PMNS matrix in leptons are named $\Theta_{Q}$ and $\Theta_{L}$, respectively. They become the orthogonal components to make a hyperbola of radius $\pi / 4$ in two-dimensional complex vector space $\mathbb{C}^{2}$. The geometric constraint is imposed by

$$
\Theta_{L}^{2}-\Theta_{Q}^{2}=\left(\frac{\pi}{4}\right)^{2}
$$

$\Theta_{Q}$ and $\Theta_{L}$ are Hermitian angles as defined in Eq. (A2) [6] and correspond to the absolute values of complex angles. With $\Theta_{Q}=0.23$ from Eq. (11), the condition is reduced to $\Theta_{L}=0.818$. Knowing a Hermitian angle $\Theta_{Q}$ leads to the direction $\left|n_{Q}\right\rangle$, and vice versa. Likewise in the Eq. (11) for the CKM matrix, $\left|n_{L}\right\rangle$ and $\Theta_{L}$ can be obtained from the parameters in the PMNS matrix.

However, taking best-fit values of the mixing angles to obtain both $\Theta_{Q}$ and $\Theta_{L}$ does not satisfy the condition in Eq. (12). For the simplest example with the best-fit values $\theta_{12}^{L}=0.59$ and $\theta_{23}^{L}=0.76$, the unitary transformation with $\delta^{L}=0$ and $\theta_{13}^{L}=0.18$ is equivalent to a 
rotation about the axis

$$
\begin{aligned}
\left|n_{L}\right\rangle & =0.82\left|o_{1}\right\rangle+0.038\left|o_{2}\right\rangle+0.57\left|o_{3}\right\rangle \\
& =0.82\left|\nu_{e}\right\rangle+0.038\left|\nu_{\mu}\right\rangle+0.57\left|\nu_{\tau}\right\rangle
\end{aligned}
$$

by an angle $\Theta_{L}=0.99$. Even though any other value of $\theta_{13}^{L}$ below the upper bound 0.23 at $3 \sigma$ CL is considered, as well as any other value of $\delta^{L}, \Theta_{L}=0.818$ cannot satisfy Eq. (12) unless values of $\theta_{12}^{L}$ and $\theta_{23}^{L}$ are far from their current best-fit values.

The uncertainties allowed by the current accuracy are given by the variations, $0.62-0.97$ for $\theta_{23}^{L}$ and $0.51-0.69$ for $\theta_{12}^{L}$ at the $3 \sigma$ CL illustrated as the width of shadow in Fig. 2. The left plot in Fig. 2 illustrates that the values of $\theta_{13}^{L}$ can satisfy the condition in Eq. (12) if $\delta^{L}=0$. Since a series of experiments on reactor neutrino oscillations, Double Chooz, Daya Bay, and RENO, aim to determine the value of $\theta_{13}^{L}$ as being larger than 0.03 in a few years [2], we can confirm a curve of the constraint in $\theta_{23}^{L}-\theta_{12}^{L}$ space. In the case where $\delta^{L}=0$, $\theta_{13}^{L}$ larger than 0.08 cannot be the physical solution, as shown in the figure. However, a $\theta_{13}^{L}$ whose curve does not pass the region of allowed data in the left figure is not ruled out.

The right plot in Fig. 2 shows that there is a certain range in the value of $\delta^{L}$ with the respect to $\theta_{13}^{L}=0.2$ which can make the constraint compatible with data within $3 \sigma$ CL. The allowed range in $\delta^{L}$ depends on the size of $\theta_{13}^{L}$ as shown in Fig. 3, so that the geometric complementarity condition predicts the $\mathrm{CP} \delta^{L}$ after $\theta_{13}^{L}$. However, the curve of $\delta^{L}=\pi$ with a smaller $\theta_{13}^{L}$ does not approach the central range of the data, even though $\theta_{13}^{L}=0$ is the closest to the center of the allowed range, because the deviation due to different $\delta^{L}$ 's is determined with the size of $\theta_{13}^{L}$ as the amplitude. It is possible that more precise measurements will rule out geometric complementarity, if they rule out $\theta_{23}^{L}<0.65$ and $\theta_{12}^{L}<0.57$.

In conclusion, complementarity and the experimental data are compatible with each other only within a small area in neutrino mixing angle parametric space. The constraint will predict the value of $\mathrm{CP} \delta^{L}$ when all the real angles are better-measured. Thus, the model with its predicted CP $\delta^{L}$ can be tested by using long base-line oscillations like JHF in the near future [7], or by using astronomical neutrino bursts [8]. 

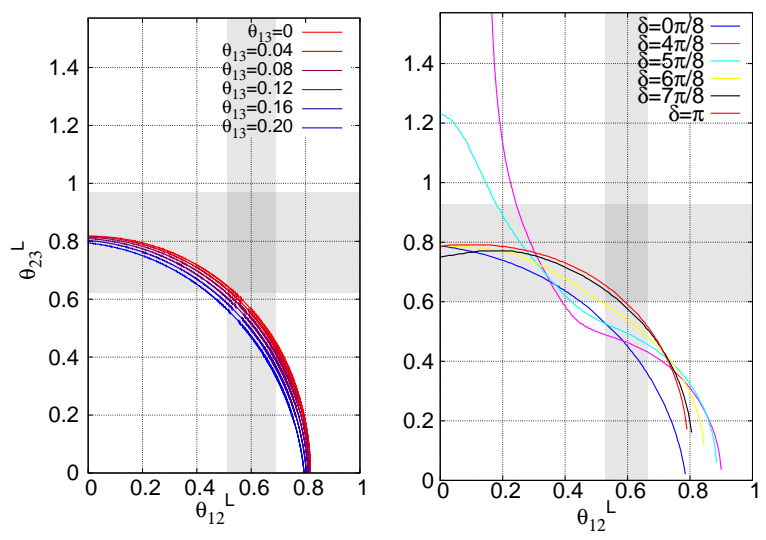

FIG. 2: Plots of $\Theta_{L}^{2}-\Theta_{Q}^{2}=(\pi / 4)^{2}$ for fixed $\delta^{L}=0$ (left) and for fixed $\theta_{13}=0.2$ (right). The dark bands indicate the global-fit ranges of $\theta_{23}$ and $\theta_{12}$ at the $3 \sigma$ level.

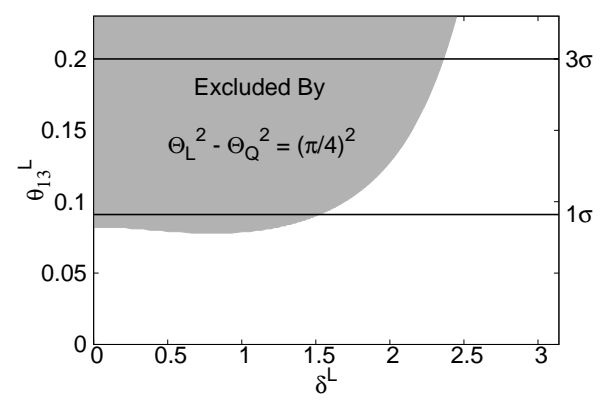

FIG. 3: The allowed value of $\delta_{C P}$ with respect to $\theta_{13}$ for the ranges in $\theta_{23}$ and $\theta_{12}$ at the $3 \sigma$ CL.

\section{APPENDIX A: COMPLEX VECTOR SPACE}

It is useful to introduce the concept of an angle in complex vector spaces [6]. In a finitedimensional real (Euclidean) vector space $\mathbb{V}_{\mathbf{R}}\left(\simeq \mathbf{R}_{n}, n \in \mathbf{N}, n \geq 2\right)$, the angle between two vectors $\mathbf{A}$ and $\mathbf{B}$ is defined in terms of the scalar product.

$$
\cos \Theta(\mathbf{A}, \mathbf{B})=\frac{(\mathbf{A}, \mathbf{B})_{\mathbf{R}}}{|\mathbf{A}||\mathbf{B}|},
$$

where $(\mathbf{A}, \mathbf{B})_{\mathbf{R}}=\sum_{k=1}^{n} A_{k} B_{k}$ and $|\mathbf{A}|=\sqrt{(\mathbf{A}, \mathbf{A})_{\mathbf{R}}}$. There are more than one definition of the angle between two vectors $\mathbf{a}$ and $\mathbf{b}$ in a finite-dimensional complex vector space $V_{\mathbf{C}}\left(\simeq \mathbf{C}_{n}, n \in \mathbf{N}, n \geq 2\right)$. A complex angle can be defined as

$$
\cos \Theta_{\mathbf{C}}(\mathbf{a}, \mathbf{b})=\frac{(\mathbf{a}, \mathbf{b})_{\mathbf{C}}}{|\mathbf{a}||\mathbf{b}|}
$$

by using the Hermitian product $(\mathbf{a}, \mathbf{b})_{\mathbf{C}}=\sum_{k=1}^{n} a_{k}^{\dagger} b_{k}$ for any pair of vectors $a, b \in \mathbb{V}_{\mathbf{C}}$. The cosine of the complex angle can be rephrased, in general, as $\cos \Theta_{\mathbf{C}}(\mathbf{a}, \mathbf{b})=\rho e^{i \varphi},(\rho \leq 1)$. It 
is useful to introduce the definitions of the Hermitian angle and the Euclidean angle, and their difference. Hermitian angle $\Theta_{\mathbf{H}}$ is defined such that

$$
\begin{aligned}
& \cos \Theta_{\mathbf{H}}(\mathbf{a}, \mathbf{b})=\left|\cos \Theta_{\mathbf{C}}(\mathbf{a}, \mathbf{b})\right|=\rho, \\
& 0 \leq \Theta_{\mathbf{H}} \leq \pi / 2
\end{aligned}
$$

where $\varphi=\varphi(\mathbf{a}, \mathbf{b}),(-\pi \leq \varphi \leq \pi)$ is called the pseudo angle of two vectors. The Euclidean angle between two vectors in $\mathbb{V}_{\mathbf{C}}$ depends on the vector space $\mathbb{V}_{\mathbf{R}}\left(\simeq \mathbf{R}_{2 n}\right)$ isometric to $\mathbb{V}_{\mathbf{C}}$ :

$$
\cos \Theta_{\mathbf{E}}(\mathbf{a}, \mathbf{b})=\cos \Theta_{\mathbf{E}}(\mathbf{A}, \mathbf{B})=\frac{(\mathbf{A}, \mathbf{B})_{\mathbf{R}}}{|\mathbf{A}||\mathbf{B}|}
$$

where the components of the vectors $\mathbf{A}, \mathbf{B}$ are related with those of $\mathbf{a}, \mathbf{b}$ in the following way: $A_{2 k-1}=\mathbf{R e} a_{k}$ and $A_{2 k}=\operatorname{Im} a_{k}, k=1 . . n$. A simple relation exists between the Hermitian angle $\Theta_{\mathbf{H}}$ and the Euclidean angle $\Theta_{\mathbf{E}}$,

$$
\cos \Theta_{\mathbf{E}}(\mathbf{a}, \mathbf{b})=\cos \Theta_{\mathbf{H}}(\mathbf{a}, \mathbf{b}) \cos \varphi
$$

\section{ACKNOWLEDGMENTS}

This research was supported by Chung-Ang University research grants in 2005.

[1] W. M. Yao et al. [Particle Data Group], J. Phys. G 33, 1 (2006).

[2] M. C. Gonzalez-Garcia and M. Maltoni, arXiv:0704.1800 [hep-ph], 2007.

[3] M. Maltoni, T. Schwetz, M. A. Tortola and J. W. F. Valle, New J. Phys. 6, 122 (2004)

[4] H. Minakata and A. Y. Smirnov, Phys. Rev. D 70, 073009 (2004) H. Minakata, arXiv:hep-ph/0505262, 2005. M. A. Schmidt and A. Y. Smirnov, Phys. Rev. D 74, 113003 (2006) S. K. Kang, C. S. Kim and J. Lee, Phys. Lett. B 619, 129 (2005)

[5] A. Strumia and F. Vissani, arXiv:hep-ph/0606054, 2006.

[6] K. Scharnhorst, Acta Appl. Math. 69, 95 (2001)

[7] Y. Itow et al. [The T2K Collaboration], arXiv:hep-ex/0106019, 2001.

[8] R. Takahashi and S. Nagataki, J. Korean Phys. Soc. 49, 1818 (2006). N. Kawanaka, S. Mineshige and S. Nagataki, J. Korean Phys. Soc. 49, 1827 (2006). K. Murase and S. Nagataki, J. Korean Phys. Soc. 49, 1834 (2006). 


\title{
A complex-angle rotation and geometric complementarity in fermion mixing
}

\author{
Kee-Hwan Nam* and Kim Siyeont \\ Department of Physics, Chung-Ang University, Seoul 156-756, Korea \\ Seungsu Hwang $\ddagger$ \\ Department of Mathematics, Chung-Ang University, Seoul 156-756, Korea
}

(Dated: today)

\begin{abstract}
The mixing among flavors in quarks or leptons in terms of a single rotation angle is defined such that three flavor eigenvectors are transformed into three mass eigenvectors by a single rotation about a common axis. We propose that a geometric complementarity condition exists between the complex angle of quarks and that of leptons in $\mathbb{C}^{2}$ space. The complementarity constraint has its rise in quark-lepton unification and is reduced to the correlation among $\theta_{12}, \theta_{23}, \theta_{13}$ and the $\mathrm{CP}$ phase $\delta$. The CP phase turns out to have a non-trivial dependence on all the other angles. We will show that further precise measurements in real angles can narrow down the allowed region of $\delta$. In comparison with other complementarity schemes, this geometric one can avoid the problem of the $\theta_{13}$ exception and can naturally keep the lepton basis being independent of quark basis.
\end{abstract}

PACS numbers: 11.30.Fs, 14.60.Pq, 14.60.St

Keywords: neutrino mixing, quark-lepton complementarity

\footnotetext{
*Electronic address: snowall@gmail.com

${ }^{\dagger}$ Electronic address: siyeon@cau.ac.kr

${ }^{\ddagger}$ Electronic address: seungsu@cau.ac.kr
} 


\section{INTRODUCTION}

With a number of successful neutrino oscillation experiments, the information on fermion masses and on the transformation between the mass basis and the weak interaction basis is getting more balanced between the quark part and the lepton part. The Cabibbo-KobayashiMaskawa(CKM) matrix of quark mixing is just a few steps from the completion. The allowed ranges of the magnitudes of the CKM elements are narrow, $\left|V_{C K M}\right|=$

$$
\left(\begin{array}{ccc}
0.97383_{-0.00023}^{+0.00024} & 0.2272_{-0.0010}^{+0.0010} & \left(3.96_{-0.09}^{+0.09}\right) \times 10^{-3} \\
0.2271_{-0.0010}^{+0.0010} & 0.97296_{-0.00024}^{+0.00024} & \left(42.21_{-0.80}^{+0.10}\right) \times 10^{-3} \\
\left(8.14_{-0.64}^{+0.32}\right) \times 10^{-3} & \left(41.61_{-0.78}^{+0.12}\right) \times 10^{-3} & 0.999100_{-0.000004}^{+0.000034}
\end{array}\right),
$$

while the ranges of the magnitudes of Potecorvo-Maki-Nakagawa-maskawa(PMNS) elements are still broad;

$$
\left|U_{P M N S}\right|=\left(\begin{array}{ccc}
0.79-0.86 & 0.50-0.61 & 0-0.20 \\
0.25-0.53 & 0.47-0.73 & 0.56-0.79 \\
0.21-0.51 & 0.42-0.69 & 0.61-0.83
\end{array}\right)
$$

at the $3 \sigma$ level [1]. The unitary transformations are conventionally described as Euler-type subsequent operations of three separate rotations,

$$
U\left(\theta_{23}, \theta_{13}, \theta_{12}, \delta\right)=R\left(\theta_{23}\right) R\left(\theta_{13}, \delta\right) R\left(\theta_{12}\right)
$$

that is, through a rotation in the $1-2$ plane, another rotation in the $1^{\prime}-3$ plane, and a third rotation in the $2^{\prime}-3^{\prime}$ plane, the mass basis is switched into the weak basis. From an analysis of the global data, the three angles in CKM have the best-fit values $\theta_{12}^{q}=0.229, \theta_{13}^{q}=0.004$, and $\theta_{23}^{q}=0.042$, and the angles in PMNS have the best-fit values $\theta_{12}^{l}=0.588, \theta_{13}^{l}=0$, and $\theta_{23}^{l}=0.756[2,3]$.

Quark-lepton complementarity (QLC) is one of the theoretical frameworks on which phenomenological data can be naturally connected to the quark-lepton unification. In many works, the QLC idea was built by the relation [4]

$$
\theta_{i j}^{q}+\theta_{i j}^{l}=\frac{\pi}{4}
$$

with the mixing angle between $\mathrm{i}$ - and $\mathrm{j}$ - generations, $\theta_{i j}$. Only $\theta_{12}$ 's and $\theta_{23}$ 's satisfy the above relation such that the complementarity gives rise to bi-maximal mixing. 
The QLC represented by Eq. (3) has a few points unexplained so far. First, like bimaximal mixing, the QLC is obliged to keep the exception with small $\theta_{13}$ 's of quarks and leptons, which cannot make the sum maximal. Second, the relation implies that the angles $\theta_{i j}^{q}$ and $\theta_{i j}^{l}$ are in a plane. In low-energy theory where the quark-lepton symmetry is broken, the common plane including those two angles requires a strong generic connection between quark bases and lepton bases in process of symmetry breaking, but there is no supporting theory.

Here, we propose a model that accommodates small $\theta_{13}$ 's and that allows $\theta_{i j}^{q}$ and $\theta_{i j}^{l}$ to belong to independent planes. In this attempt, the transformation from the weak basis to the mass basis can be obtained by a single complex-angle rotation about a properly defined axis [5]. In other words, by a single rotation about an axis, the weak eigenstates $\left(\nu_{e}, \nu_{\mu}, \nu_{\tau}\right)$ or $(d, s, b)$ are switched into $\left(\nu_{1}, \nu_{2}, \nu_{3}\right)$ or $\left(d_{1}, d_{2}, d_{3}\right)$, respectively. Thus, there are two complex angles, one corresponding to quark mixing and the other corresponding to lepton mixing. We introduce the complementarity by a relation of those two complex angles such that

$$
\Theta_{L}^{2}-\Theta_{Q}^{2}=\left(\frac{\pi}{4}\right)^{2}
$$

where they are the orthogonal components of a hyperbola of radius $\pi / 4$. With such a geometric constraint, the model can protect the complementarity from the $\theta_{13}$ exception. Furthermore, the quark basis and the lepton basis are independent of each others as implied by completely broken quark-lepton symmetry.

In Section II, the definition of the complex angle and the axis to represent the transformation will be introduced. In Section III, using the hyperbolic condition, the allowed range of Dirac Charge-Parity violating $(\mathrm{CP})$ phase will be predicted. It will be shown that the more precise measurements in other angles in the future can test the model itself, as well as the $\mathrm{CP}$ violation testable in neutrino oscillation. Only the CKM-type matrix without Majorana phases is considered as the PMNS matrix. An extended work with more details, including Majorana phases and the physical implication relevant to them, is in progress in other work. A brief on the convention to deal with complex angles is attached. 


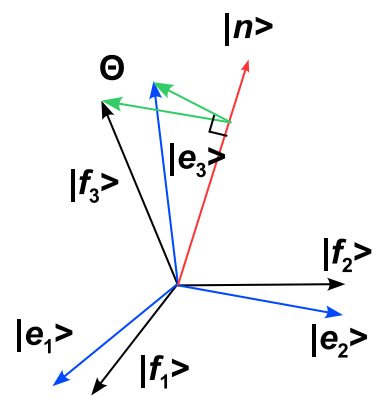

FIG. 1: The rotation of $\Theta$ about $\mid n>$ transforms $\mid e_{i}>$ 's into $\mid f_{\alpha}>$ 's.

\section{A SINGLE COMPLEX-ANGLE ROTATION}

In both quarks and leptons, a unitary transformation between flavor eigenstates $\left|f_{\alpha}\right\rangle$ and mass eigenstates $\left|e_{i}\right\rangle$ consists minimally of three angles and a CP phase:

$$
\left|f_{\alpha}\right\rangle=U\left(\theta_{12}, \theta_{13}, \theta_{23}, \delta\right)\left|e_{i}\right\rangle
$$

If the weak interaction basis is properly chosen such that the mass matrix of up-type quarks and that of charged leptons are diagonal, $\left|f_{\alpha}\right\rangle$ and $U$ in Eq. (5) represent either down-type quarks and the CKM matrix in the quark sector or neutrinos and the PMNS matrix in the lepton sector. Suppose, in a representation, that $\left|e_{1}\right\rangle=(1,0,0)^{T},\left|e_{2}\right\rangle=(0,1,0)^{T}$, and $\left|e_{3}\right\rangle=(0,0,1)^{T}$. Then $\left|f_{\alpha}\right\rangle=\left(U_{f_{\alpha} 1}, U_{f_{\alpha} 2}, U_{f_{\alpha} 3}\right)^{T}$, for $f_{\alpha}=d, s, b$ or $f_{\alpha}=e, \mu, \tau$. The $U_{f_{\alpha} i}$ is

an element of the transformation matrix in Eq. (5). The components of $f_{\alpha}$ are denoted by $\left(f_{1}, f_{2}, f_{3}\right)$ if the specification of 'quark or lepton' is not necessary. In a real vector space, an orthogonal set of three vectors can fit into another orthogonal set of three vectors simply by rotating the original set about a common axis, which can be found to be invariant under the rotation. Likewise, the unitary transformation in Eq. (5) can be replaced by a rotation with a single complex angle.

If one constructs a vector $|n\rangle$ as the axis of the rotation, the rotation angle of a vector $\left|e_{i}\right\rangle$ about $|n\rangle$ is the same as the angle between the following two vectors $\left|e_{i}^{\perp}\right\rangle$ and $\left|f_{\alpha}^{\perp}\right\rangle$ that are orthogonal to $|n\rangle$ :

$$
\begin{aligned}
\cos \Theta & =\frac{\left\langle e_{i}^{\perp} \mid f_{\alpha}^{\perp}\right\rangle}{\sqrt{\left\langle e_{i}^{\perp} \mid e_{i}^{\perp}\right\rangle} \sqrt{\left\langle f_{\alpha}^{\perp} \mid f_{\alpha}^{\perp}\right\rangle}} \\
& =\frac{\left\langle e_{i} \mid f_{\alpha}\right\rangle-\left\langle e_{i} \mid n\right\rangle\left\langle n \mid f_{\alpha}\right\rangle}{\sqrt{\left\langle e_{i}^{\perp} \mid e_{i}^{\perp}\right\rangle} \sqrt{\left\langle f_{\alpha}^{\perp} \mid f_{\alpha}^{\perp}\right\rangle}},
\end{aligned}
$$


where

$$
\begin{aligned}
& \left|f_{\alpha}^{\perp}\right\rangle=\left|f_{\alpha}\right\rangle-|n\rangle\left\langle n \mid f_{\alpha}\right\rangle, \\
& \left|e_{i}^{\perp}\right\rangle=\left|e_{i}\right\rangle-|n\rangle\left\langle n \mid e_{i}\right\rangle .
\end{aligned}
$$

The rotation axis $|n\rangle=\left(n_{x}, n_{y}, n_{z}\right)^{T}$ has the same components on the mass basis $\left|e_{i}\right\rangle$ as on the flavor basis $\left|f_{\alpha}\right\rangle$, that is,

$$
n_{x}\left|e_{1}\right\rangle+n_{y}\left|e_{2}\right\rangle+n_{z}\left|e_{3}\right\rangle=n_{x}\left|f_{1}\right\rangle+n_{y}\left|f_{2}\right\rangle+n_{z}\left|f_{3}\right\rangle
$$

which is a normalized vector with $\left|n_{x}\right|^{2}+\left|n_{y}\right|^{2}+\left|n_{z}\right|^{2}=1$. Substituting Eq. (5) into Eq. (7) results in the following combined equations:

$$
\begin{aligned}
& c_{13} c_{12} n_{x}-c_{13} s_{12} n_{y}+s_{13} e^{-i \delta} n_{z}=n_{x} \\
& \left(-c_{23} s_{12}-s_{23} s_{13} c_{12} e^{i \delta}\right) n_{x}+\left(c_{23} c_{12}-s_{23} s_{13} s_{12} e^{i \delta}\right) n_{y}+s_{23} c_{13} n_{z}=n_{y} \\
& \left(s_{23} s_{12}-c_{23} s_{13} c_{12} e^{i \delta}\right) n_{x}+\left(-s_{23} c_{12}-c_{23} s_{13} s_{12} e^{i \delta}\right) n_{y}+s_{23} c_{13} n_{z}=n_{z} .
\end{aligned}
$$

It is possible to express $|n\rangle$ immediately in terms of mixing parameters. For instance, $\left|e_{1}^{\perp}\right\rangle,\left|f_{1}^{\perp}\right\rangle$, the projected vectors of $\left|e_{1}\right\rangle,\left|f_{1}\right\rangle$ on the plane perpendicular to $|n\rangle$, are, according to Eq. (6),

$$
\begin{aligned}
&\left|e_{1}^{\perp}\right\rangle=\left(\begin{array}{c}
1-\left|n_{x}\right|^{2} \\
-n_{x}^{*} n_{y} \\
-n_{x}^{*} n_{z}
\end{array}\right), \\
&\left|f_{1}^{\perp}\right\rangle=\left(\begin{array}{c}
U_{11} \\
U_{12} \\
U_{13}
\end{array}\right)-\left(n_{x}^{*} U_{11}+n_{y}^{*} U_{12}+n_{z}^{*} U_{13}\right)\left(\begin{array}{c}
n_{x} \\
n_{y} \\
n_{z}
\end{array}\right) .
\end{aligned}
$$

Then, the $\cos \Theta$ in Eq. (마) reduces to

$$
\cos \Theta=\frac{U_{11}-n_{x}\left(n_{x}^{*} U_{11}+n_{y}^{*} U_{12}+n_{z}^{*} U_{13}\right)}{\left.\sqrt{\left(1-\left|n_{x}\right|^{2}\right)\left(1-\left|n_{x}^{*} U_{11}+n_{y}^{*} U_{12}+n_{z}^{*} U_{13}\right|^{2}\right.}\right)},
$$

because $\left(n_{x}, n_{y}, n_{z}\right)$ is obtained in terms of mixing angles and a phase, as is $\cos \Theta$.

The four physical parameters in the unitary mixing in Eq. (5) are now all embedded in the direction of $|n\rangle$. For a complex vector, one can remove the imaginary phase in one of the 
elements by multiplying all the elements in the vector by an overall phase factor. Together with normalization, the complex vector $|n\rangle=\left(n_{x}, n_{y}, n_{z}\right)^{T}$ does include just four independent parameters while the complex angle $\Theta$ does not include any additional independent parameter. Once $|n\rangle$ is found, then a unique $\Theta$ is determined to represent the transformation. It can be also checked that $\cos \Theta$ is complex unless $\sin \delta$ vanishes. As an example to find $|n\rangle$ and $\Theta$, if one considers $\theta_{12}=0.23, \theta_{23}=0.042, \theta_{13}=0.004$, and $\delta=0.99$ induced from the best-fit values of the elements in the CKM matrix, the rotation of an angle $\Theta=0.23 e^{i \varphi}, \varphi<0.01$, obtained from Eq. (10) about the axis

$$
\begin{aligned}
\left|n_{Q}\right\rangle & =0.18 e^{-.005 i}\left|e_{1}\right\rangle+0.017 e^{0.868 i}\left|e_{2}\right\rangle+0.983\left|e_{3}\right\rangle \\
& =0.18 e^{-.005 i}|d\rangle+0.017 e^{0.868 i}|s\rangle+0.983|b\rangle
\end{aligned}
$$

results in the same transformation as the CKM matrix does.

\section{HYPERBOLIC COMPLEMENTARITY CONDITION IN $\mathbb{C}^{2}$ SPACE}

As shown in the previous section, there exists a single complex angle rotation that replaces any three-dimensional unitary transformation. The single complex angles to replace CKM matrix in quarks and PMNS matrix in leptons are named $\Theta_{Q}$ and $\Theta_{L}$, respectively. They become the orthogonal components to make a hyperbola of radius $\pi / 4$ in two-dimensional complex vector space $\mathbb{C}^{2}$. The geometric constraint is imposed by

$$
\Theta_{L}^{2}-\Theta_{Q}^{2}=\left(\frac{\pi}{4}\right)^{2}
$$

$\Theta_{Q}$ and $\Theta_{L}$ are Hermitian angles as defined in Eq. (A2) [6] and correspond to the absolute values of complex angles. With $\Theta_{Q}=0.23$ from Eq. (11), the condition is reduced to $\Theta_{L}=0.818$. Knowing a Hermitian angle $\Theta_{Q}$ leads to the direction $\left|n_{Q}\right\rangle$, and vice versa. Likewise in the Eq. (11) for the CKM matrix, $\left|n_{L}\right\rangle$ and $\Theta_{L}$ can be obtained from the parameters in the PMNS matrix.

However, taking best-fit values of the mixing angles to obtain both $\Theta_{Q}$ and $\Theta_{L}$ does not satisfy the condition in Eq. (12). For the simplest example with the best-fit values $\theta_{12}^{L}=0.59$ and $\theta_{23}^{L}=0.76$, the unitary transformation with $\delta^{L}=0$ and $\theta_{13}^{L}=0.18$ is equivalent to a 
rotation about the axis

$$
\begin{aligned}
\left|n_{L}\right\rangle & =0.82\left|o_{1}\right\rangle+0.038\left|o_{2}\right\rangle+0.57\left|o_{3}\right\rangle \\
& =0.82\left|\nu_{e}\right\rangle+0.038\left|\nu_{\mu}\right\rangle+0.57\left|\nu_{\tau}\right\rangle
\end{aligned}
$$

by an angle $\Theta_{L}=0.99$. Even though any other value of $\theta_{13}^{L}$ below the upper bound 0.23 at $3 \sigma$ CL is considered, as well as any other value of $\delta^{L}, \Theta_{L}=0.818$ cannot satisfy Eq. (12) unless values of $\theta_{12}^{L}$ and $\theta_{23}^{L}$ are far from their current best-fit values.

The uncertainties allowed by the current accuracy are given by the variations, $0.62-0.97$ for $\theta_{23}^{L}$ and $0.51-0.69$ for $\theta_{12}^{L}$ at the $3 \sigma$ CL illustrated as the width of shadow in Fig. 2. The left plot in Fig. 2 illustrates that the values of $\theta_{13}^{L}$ can satisfy the condition in Eq. (12) if $\delta^{L}=0$. Since a series of experiments on reactor neutrino oscillations, Double Chooz, Daya Bay, and RENO, aim to determine the value of $\theta_{13}^{L}$ as being larger than 0.03 in a few years [2], we can confirm a curve of the constraint in $\theta_{23}^{L}-\theta_{12}^{L}$ space. In the case where $\delta^{L}=0$, $\theta_{13}^{L}$ larger than 0.08 cannot be the physical solution, as shown in the figure. However, a $\theta_{13}^{L}$ whose curve does not pass the region of allowed data in the left figure is not ruled out.

The right plot in Fig. 2 shows that there is a certain range in the value of $\delta^{L}$ with the respect to $\theta_{13}^{L}=0.2$ which can make the constraint compatible with data within $3 \sigma$ CL. The allowed range in $\delta^{L}$ depends on the size of $\theta_{13}^{L}$ as shown in Fig. 3, so that the geometric complementarity condition predicts the $\mathrm{CP} \delta^{L}$ after $\theta_{13}^{L}$. However, the curve of $\delta^{L}=\pi$ with a smaller $\theta_{13}^{L}$ does not approach the central range of the data, even though $\theta_{13}^{L}=0$ is the closest to the center of the allowed range, because the deviation due to different $\delta^{L}$ 's is determined with the size of $\theta_{13}^{L}$ as the amplitude. It is possible that more precise measurements will rule out geometric complementarity, if they rule out $\theta_{23}^{L}<0.65$ and $\theta_{12}^{L}<0.57$.

In conclusion, complementarity and the experimental data are compatible with each other only within a small area in neutrino mixing angle parametric space. The constraint will predict the value of $\mathrm{CP} \delta^{L}$ when all the real angles are better-measured. Thus, the model with its predicted CP $\delta^{L}$ can be tested by using long base-line oscillations like JHF in the near future [7], or by using astronomical neutrino bursts [8]. 

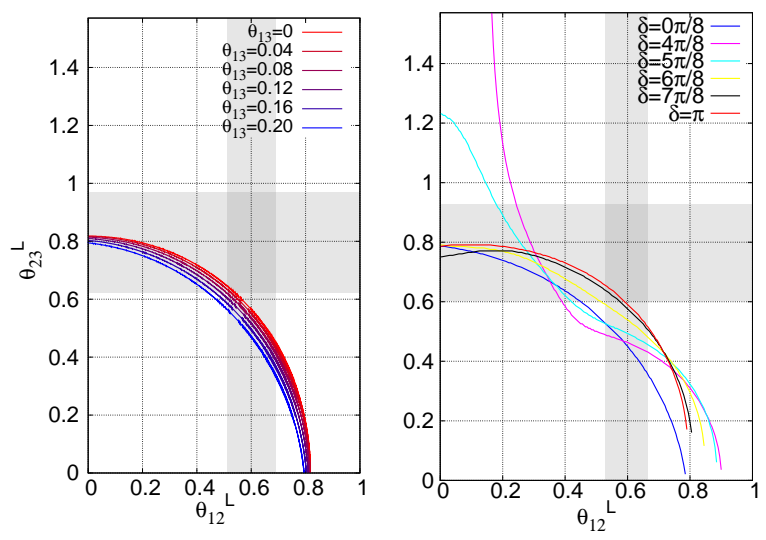

FIG. 2: Plots of $\Theta_{L}^{2}-\Theta_{Q}^{2}=(\pi / 4)^{2}$ for fixed $\delta^{L}=0$ (left) and for fixed $\theta_{13}=0.2$ (right). The dark bands indicate the global-fit ranges of $\theta_{23}$ and $\theta_{12}$ at the $3 \sigma$ level.

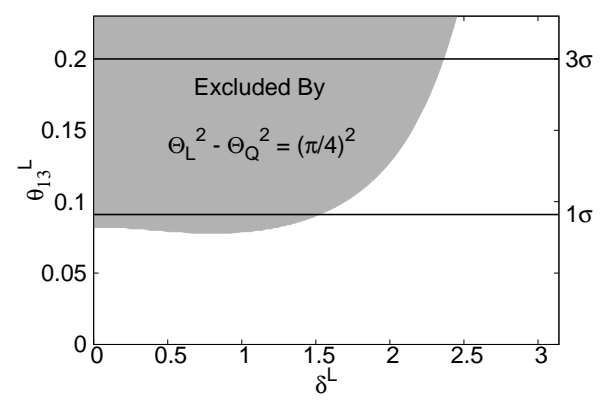

FIG. 3: The allowed value of $\delta_{C P}$ with respect to $\theta_{13}$ for the ranges in $\theta_{23}$ and $\theta_{12}$ at the $3 \sigma$ CL.

\section{APPENDIX A: COMPLEX VECTOR SPACE}

It is useful to introduce the concept of an angle in complex vector spaces [6]. In a finitedimensional real (Euclidean) vector space $\mathbb{V}_{\mathbf{R}}\left(\simeq \mathbf{R}_{n}, n \in \mathbf{N}, n \geq 2\right)$, the angle between two vectors $\mathbf{A}$ and $\mathbf{B}$ is defined in terms of the scalar product.

$$
\cos \Theta(\mathbf{A}, \mathbf{B})=\frac{(\mathbf{A}, \mathbf{B})_{\mathbf{R}}}{|\mathbf{A}||\mathbf{B}|},
$$

where $(\mathbf{A}, \mathbf{B})_{\mathbf{R}}=\sum_{k=1}^{n} A_{k} B_{k}$ and $|\mathbf{A}|=\sqrt{(\mathbf{A}, \mathbf{A})_{\mathbf{R}}}$. There are more than one definition of the angle between two vectors $\mathbf{a}$ and $\mathbf{b}$ in a finite-dimensional complex vector space $V_{\mathbf{C}}\left(\simeq \mathbf{C}_{n}, n \in \mathbf{N}, n \geq 2\right)$. A complex angle can be defined as

$$
\cos \Theta_{\mathbf{C}}(\mathbf{a}, \mathbf{b})=\frac{(\mathbf{a}, \mathbf{b})_{\mathbf{C}}}{|\mathbf{a}||\mathbf{b}|}
$$

by using the Hermitian product $(\mathbf{a}, \mathbf{b})_{\mathbf{C}}=\sum_{k=1}^{n} a_{k}^{\dagger} b_{k}$ for any pair of vectors $a, b \in \mathbb{V}_{\mathbf{C}}$. The cosine of the complex angle can be rephrased, in general, as $\cos \Theta_{\mathbf{C}}(\mathbf{a}, \mathbf{b})=\rho e^{i \varphi},(\rho \leq 1)$. It 
is useful to introduce the definitions of the Hermitian angle and the Euclidean angle, and their difference. Hermitian angle $\Theta_{\mathbf{H}}$ is defined such that

$$
\begin{aligned}
& \cos \Theta_{\mathbf{H}}(\mathbf{a}, \mathbf{b})=\left|\cos \Theta_{\mathbf{C}}(\mathbf{a}, \mathbf{b})\right|=\rho, \\
& 0 \leq \Theta_{\mathbf{H}} \leq \pi / 2
\end{aligned}
$$

where $\varphi=\varphi(\mathbf{a}, \mathbf{b}),(-\pi \leq \varphi \leq \pi)$ is called the pseudo angle of two vectors. The Euclidean angle between two vectors in $\mathbb{V}_{\mathbf{C}}$ depends on the vector space $\mathbb{V}_{\mathbf{R}}\left(\simeq \mathbf{R}_{2 n}\right)$ isometric to $\mathbb{V}_{\mathbf{C}}$ :

$$
\cos \Theta_{\mathbf{E}}(\mathbf{a}, \mathbf{b})=\cos \Theta_{\mathbf{E}}(\mathbf{A}, \mathbf{B})=\frac{(\mathbf{A}, \mathbf{B})_{\mathbf{R}}}{|\mathbf{A}||\mathbf{B}|}
$$

where the components of the vectors $\mathbf{A}, \mathbf{B}$ are related with those of $\mathbf{a}, \mathbf{b}$ in the following way: $A_{2 k-1}=\mathbf{R e} a_{k}$ and $A_{2 k}=\operatorname{Im} a_{k}, k=1 . . n$. A simple relation exists between the Hermitian angle $\Theta_{\mathbf{H}}$ and the Euclidean angle $\Theta_{\mathbf{E}}$,

$$
\cos \Theta_{\mathbf{E}}(\mathbf{a}, \mathbf{b})=\cos \Theta_{\mathbf{H}}(\mathbf{a}, \mathbf{b}) \cos \varphi
$$

\section{ACKNOWLEDGMENTS}

This research was supported by Chung-Ang University research grants in 2005.

[1] W. M. Yao et al. [Particle Data Group], J. Phys. G 33, 1 (2006).

[2] M. C. Gonzalez-Garcia and M. Maltoni, arXiv:0704.1800 [hep-ph], 2007.

[3] M. Maltoni, T. Schwetz, M. A. Tortola and J. W. F. Valle, New J. Phys. 6, 122 (2004)

[4] H. Minakata and A. Y. Smirnov, Phys. Rev. D 70, 073009 (2004) H. Minakata, arXiv:hep-ph/0505262, 2005. M. A. Schmidt and A. Y. Smirnov, Phys. Rev. D 74, 113003 (2006) S. K. Kang, C. S. Kim and J. Lee, Phys. Lett. B 619, 129 (2005)

[5] A. Strumia and F. Vissani, arXiv:hep-ph/0606054, 2006.

[6] K. Scharnhorst, Acta Appl. Math. 69, 95 (2001)

[7] Y. Itow et al. [The T2K Collaboration], arXiv:hep-ex/0106019, 2001.

[8] R. Takahashi and S. Nagataki, J. Korean Phys. Soc. 49, 1818 (2006). N. Kawanaka, S. Mineshige and S. Nagataki, J. Korean Phys. Soc. 49, 1827 (2006). K. Murase and S. Nagataki, J. Korean Phys. Soc. 49, 1834 (2006). 